\title{
Accelerometers in collars identify behavioral states in captive African elephants Loxodonta africana
}

\author{
Joseph Soltis $^{1, *}$, Rory P. Wilson ${ }^{2}$, Iain Douglas-Hamilton ${ }^{3,4}$, Fritz Vollrath ${ }^{3,4}$, \\ Lucy E. King ${ }^{3}$, Anne Savage ${ }^{1}$ \\ ${ }^{1}$ Education and Science, Disney's Animal Kingdom ${ }^{\circledR}$, Florida 32830, USA \\ ${ }^{2}$ Biological Sciences, Institute of Environmental Sustainability, Swansea University, Swansea SA2 8PP, UK \\ ${ }^{3}$ Save the Elephants, PO Box 54667, Nairobi 00200, Kenya \\ ${ }^{4}$ Animal Behaviour Research Group, Department of Zoology, University of Oxford, Oxford OX1 2JD, UK
}

\begin{abstract}
Accelerometers are motion-detection devices that, when attached to animals, are capable of detecting body orientation, overall activity levels, and specific behavior patterns. We deployed accelerometers in order to study the hypothesis that accelerometer output would allow us to distinguish between 4 behavior patterns in 3 adult female African elephants Loxodonta africana at Disney's Animal Kingdom ${ }^{\circledR}$, Florida, USA. Tri-axial accelerometer data loggers were attached to the tops of collars worn around the elephants' necks. Behavior was documented on video while the accelerometer output was stored on the data logger. Feeding, bathing, and walking behaviors were recorded in all 3 subjects, while swaying behavior could be recorded in only 1 subject. Data in the 3 physical dimensions (sway, surge, and heave) were analyzed in terms of overall magnitude of movement (dynamic acceleration) and in terms of the periodicity of movement. When classifying accelerometer data of unlabeled origin to the correct behavioral state, overall success ranged from 70 to $91 \%$. Bathing was sometimes confused with feeding and walking, but feeding, walking, and swaying were easily distinguished from each other. These results show that data from accelerometers can distinguish an elephant's behavioral states, and thus may be used to monitor elephant behavior remotely. Such devices could be deployed for a variety of purposes, ranging from monitoring elephant activity in zoos to an early-warning system that could alert the authorities when wild elephants are being illegally hunted.
\end{abstract}

KEY WORDS: Behavior measurement $\cdot$ Accelerometry $\cdot$ Elephant management Resale or republication not permitted without written consent of the publisher

\section{INTRODUCTION}

The use of accelerometers to remotely measure animal behavior has increased in recent years (e.g. Godfrey et al. 2008, Shepard et al. 2008, Sala et al. 2011). When attached to animals, these devices can record acceleration due to animal orientation and movement, so that animal behavior can be inferred from the accelerometer data alone. Accelerometers have been extremely useful in measuring behavior in circumstances difficult for human observation, such as in aquatic and aerial environments. Even in terres- trial species, accelerometers can be used to record animal behavior automatically without human presence. Such devices have been employed to remotely measure animal walking distance (Rothwell et al. 2011), animal orientation (Lyons et al. 2005, Ringgenberg et al. 2010), and animal activity level and metabolic rate (Wilson et al. 2006, 2008, Halsey et al. 2009, Enstipp et al. 2011).

When animals engage in specific behaviors such as walking, swimming, or flying, the associated movements may result in signature patterns of acceleration in the 3 physical dimensions. Thus, accelerometers 
placed onto the appropriate body parts of animal subjects can be used to identify specific behaviors. For example, accelerometers have been used to differentiate between standing, sitting, floating, walking, flying, and diving in imperial cormorants (Gómez-Laich et al. 2008), standing, lying down, ruminating, feeding, normal walking, and lame walking in dairy cows (Martiskainen et al. 2009), resting, walking, grazing, and browsing in goats (Moreau et al. 2009), feeding and breathing in loggerhead sea turtles (Okuyama et al. 2009), and swimming and mating in nurse sharks (Whitney et al. 2010).

The development of a system to collect and analyze accelerometer data from elephants could be a powerful tool for monitoring elephant behavior in the wild as well as for zoo management. In the wild, GPS and accelerometer data could potentially be used to filter and classify behavior patterns that in turn trigger immediate wireless transmissions to appropriate personnel who could be guided to the location of the elephant. For example, if accelerometer data indicated signs of physical distress such as unusually high or low activity or limping, then electronic alerts could be sent to conservation biologists and wildlife veterinarians. Indeed, it has already been shown for dogs and dairy cattle that accelerometers can distinguish normal from impaired walking (Barthélémy et al. 2009, Chapinal et al. 2011). Additionally, if behavior patterns associated with human-elephant conflict could be characterized (e.g. head up, charging, fleeing), then electronic messages could be sent to wildlife veterinarians and rapid-response anti-poaching units in real time. Such devices would greatly reduce the lag time between the onset of elephant distress or injury and the delivery of aid or deployment of law enforcement personnel.

Accelerometer data may also become a useful tool for the management of captive elephants. For example, it is thought that lack of exercise in zoo elephants may contribute to health problems such as osteomyelitis (e.g. Gage 2001). Accelerometer data could be coded into daily reports for animal managers and veterinarians that include activity levels and rate of energy expenditure (e.g. Wilson et al. 2008). Additionally, the amount of time elephants engage in specific behaviors such as walking, feeding, playing, fighting, or swaying could be documented for caregivers.

The goal of this study was to determine whether accelerometer data can effectively distinguish between several common elephant behaviors, expanding on work by Rothwell et al. (2011) who placed accelerometers in anklets that measured elephant walking distance. Remote sensing devices such as
GPS units have traditionally been attached to elephants by placing collars around their necks (e.g. Wall et al. 2006). Similarly, at Disney's Animal Kingdom ${ }^{\circledR}$, we have developed GPS- and audio-recording collars that elephants routinely wear around their necks as tools in scientific studies (e.g. Soltis et al. 2005, 2011, Leighty et al. 2008). Collars are the chief means of attaching recording devices to wild elephants and thus provide the obvious means of 'truthing' the reliability and accuracy of accelerometer data before any deployment in the wild. We attached tri-axial accelerometers to the tops of collars that elephants routinely wear around their necks at Disney's Animal Kingdom ${ }^{\circledR}$. We sought to determine whether data generated by the accelerometers could distinguish between 4 behavior patterns: feeding, bathing, walking, and swaying.

\section{MATERIALS AND METHODS}

\section{Subjects and housing}

The social group under study was housed at Disney's Animal Kingdom ${ }^{\circledR}$, Florida, USA, in a 1.64 ha outdoor exhibit (for details see Leighty et al. 2009). The naturalistic outdoor enclosure contained a pool in which multiple adult elephants can fully submerge, a mud wallow, multiple scratching surfaces, and large rock rings that provide areas for animals to move out of the visual range of other animals. The social group included 3 adult females (31, 27, and 25 yr old) and their 3 female offspring $(2.5 \mathrm{mo}, 5 \mathrm{yr}$, and $6 \mathrm{yr})$. One non-related male $(7 \mathrm{yr}$ ) was rotated into the social group on a regular basis for socialization purposes, bringing the maximum group size to 7 individuals. Data presented here were collected from the 3 adult females between 10 June and 30 December 2011.

\section{Accelerometer and behavioral data collection}

Tri-axial accelerometers (X9-2mini, Gulf Coast Data Concepts) were attached to the existing audioand GPS-recording collars that the elephants routinely wear around their necks. These collars are described in detail in Leighty et al. (2009). Accelerometers $(0.8 \times 2.5 \times 6.5 \mathrm{~cm} ; 17.5 \mathrm{~g})$ were housed on top of the collar such that the devices rested on top of the elephants' neck (Fig. 1). The device was inserted into a waterproof housing, consisting of an enclosed PVC pipe braced inside a short length of fire hose. The accelerometer housing was bolted to the existing 


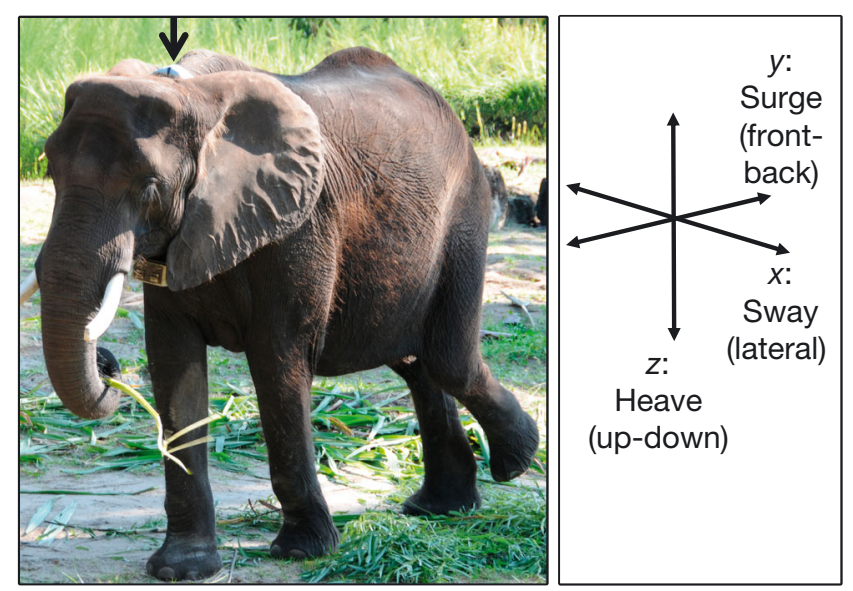

Fig. 1. Loxodonta africana. Accelerometer orientation on the elephants. Left panel shows an adult female elephant wearing the collar with the accelerometer housing positioned on top of the neck (black arrow). Right panel shows the orientation of the 3 axes in the accelerometer

collar such that the device could not move independently of the collar. The accelerometer and casing materials added $350 \mathrm{~g}$ to the existing 4.0 to $4.5 \mathrm{~kg}$ audio- and GPS-recording collars. The orientation of the device was as follows: the $x$-axis registered sway (lateral, i.e. left-right acceleration), the $y$-axis registered surge (anterior-posterior, i.e. front-back acceleration), and the $z$-axis registered heave (dorsalventral, i.e. up-down acceleration; see Fig. 1). The accelerometer was programmed to collect data at a $320 \mathrm{~Hz}$ sample rate with a 16 -bit resolution for $7 \mathrm{~h}$ (to bracket filming times).

To validate the accelerometer data against actual behavior, we videotaped elephants in their outdoor habitat while accelerometers were recording (except for swaying, which was filmed in the elephant barn before release into the outdoor enclosure). Time from a hand-held GPS device (Garmin Rino 530) was used to set the timer on the accelerometer and to timestamp the video with an audio cue, so that the accelerometer and video data were synchronized. In these analyses, we chose 4 active behaviors that were either common or important elephant behaviors, and that occurred in long bouts: walking (subject moves at any speed), feeding (subject collects food from the ground with the trunk, delivers food to the mouth with the trunk, and consumes the food, while standing), bathing (subject collects mud or water with the trunk, and uses trunk to apply the mud or water to various parts of the body, while standing), and swaying (subject swings head back and forth laterally in a repetitive, stereotypical manner, while standing).

\section{Accelerometer data measurement}

Total acceleration is traditionally divided into 2 components: static and dynamic acceleration (e.g. Shepard et al. 2008). Static acceleration refers to the $g$-forces due to the overall orientation of the device with respect to the gravitational field. For example, when the accelerometer device is flat and still, then the $x$-axis (sway) and the $y$-axis (surge) will read 0 , while the $z$-axis (heave) will read -1.0, the latter reflecting the force of gravity. When the device is oriented at a different angle but remains still, the force of gravity will act on a different axis or be distributed across 2 or 3 axes, depending on the exact orientation of the device. This orientation is the static acceleration. Dynamic acceleration refers to the $g$-forces due to movement of the device.

Dynamic acceleration can be obtained by subtracting the static acceleration from the total acceleration, and we performed these calculations in Origin software (version 8.5.1, Originlab) following Shepard et al. (2008). First, the total acceleration data stream was smoothed using a $2 \mathrm{~s}$ window to obtain the static acceleration component. Next, the static acceleration values were subtracted from the total acceleration values to obtain the dynamic acceleration. The resulting data correct for orientation of the device on the animal, and are centered on 0 . Deviations from 0 indicate the magnitude of acceleration due to movement of the device. Measurement of dynamic acceleration was computed by taking the mean of the absolute values of the dynamic acceleration data. Fig. 2 shows examples of dynamic acceleration in the 3 axes for the 4 elephant behaviors.

Measures of dynamic acceleration are ideal for determining the magnitude of movement, but they are not as useful for quantifying the periodicity of movement. For example, Fig. 2 shows that walking and swaying behavior show periodic oscillations, while feeding and bathing show little to no such periodicity. Time-series data analyses are useful for examining such periodicity, and have been previously employed to examine accelerometer data (Watanabe et al. 2005, Sakamoto et al. 2009). We have made extensive use of such time-series analyses in our acoustics research on elephants (e.g. Soltis et al. 2011) and employed those techniques here to examine this aspect of the data.

Processing of accelerometer data for time-series analysis proceeded as follows. First, the total acceleration data were transformed into acoustic (.wav) files in Adobe Audition (version 2.0, Adobe Systems), after which analyses were conducted in PRAAT (version 4.5.18; Boersma \& Weenink 2007) using fully 


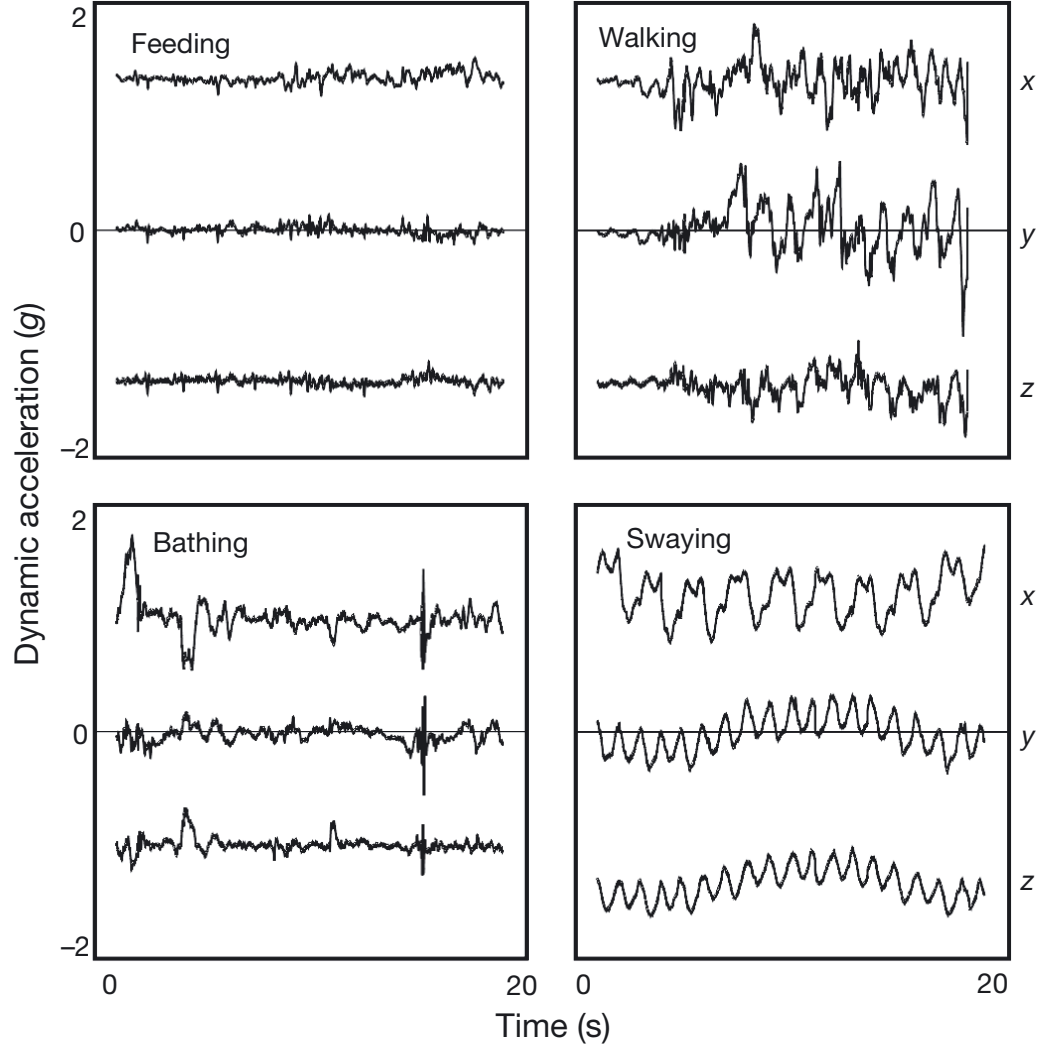

Fig. 2. Loxodonta africana. Dynamic acceleration for 4 behaviors from 1 adult female. Data for all 3 axes are shown ( $x$ : sway; $y$ : surge; $z$ : heave). The $x$ - and $z$-axes are offset by +1 and -1 , respectively, to simultaneously visualize data from all 3 axes

automated routines (see Soltis et al. 2011 for examples of these techniques used on acoustic data). First, the signal was low-pass filtered at $5 \mathrm{~Hz}$ (Hanning window, $2 \mathrm{~Hz}$ smoothing), down-sampled to a $10 \mathrm{~Hz}$ sample rate, and a Fourier transform was performed. Noisy elements in the low frequencies $(<0.35 \mathrm{~Hz})$ were present in most signals, and periodicity was observed in frequencies below $1.5 \mathrm{~Hz}$, so data from the Fourier spectrum between 0.35 and $1.50 \mathrm{~Hz}$ were analyzed. The maximum amplitude and standard deviation of the resulting spectrum were calculated, with higher values indicating higher degrees of periodicity. To determine the absolute period, the frequency with the highest amplitude (peak frequency) was calculated. Fig. 3 shows examples of the Fourier transforms for the 4 behaviors examined here.

\section{Data analysis}

We attempted to obtain ten $20 \mathrm{~s}$ sequences of each behavior from each of 3 females, for a total of 30 sequences for each behavior, which would give suffi- cient power for our statistical tests (see below). Sequences of behavior that did not last a full $20 \mathrm{~s}$ were not used. We obtained the target number of sequences from each female for feeding, bathing, and walking, but we could only obtain swaying behavior from 1 female. This female swayed at predictable times on most days, but the other 2 females were rarely or never observed swaying (keeper observations), did not sway at predictable times of the day, and were not observed swaying during any of the observation periods of the study. Therefore, we obtained 30 swaying sequences only from the one female.

The behavioral measures described above were conducted on these 120 data sequences and analyzed using SPSS software (version 15.0). We performed discriminant function analysis (DFA) in order to determine whether these measures of accelerometer data could be used to classify unlabeled behaviors into the correct behavioral state (Quinn \& Keough 2002). Data were transformed and processed before conducting the DFA so as not to violate data assumptions. First, the distribution of many variables exhibited right-skewed outliers, so all variables were $\log _{10}$ transformed, which increased normality. Second, many of the measurements were highly correlated so we performed principal components analysis (PCA) prior to DFA, to reduce the number of original variables to a smaller number of uncorrelated factors. Factors with eigenvalues $>1$ were retained and Varimax-rotated, and these factors were entered into the DFA in place of the original variables. Third, homogeneity of variance assumptions were not violated, as the PCA factor scores exhibited almost identical variances.

For classification in the DFA, we used the 'leaveone-out' technique, in which 1 behavioral sequence of the 120 sequences is left out of each analysis. The left-out sequence is then classified into 1 of the 4 behavioral states based on the functions derived from the other sequences, and is scored as either correctly or incorrectly classified. This process is repeated for every behavioral sequence, so that an overall classification success percentage can be calculated. In the leave-one-out classification, training and test data are from the same individuals. In a more conserva- 

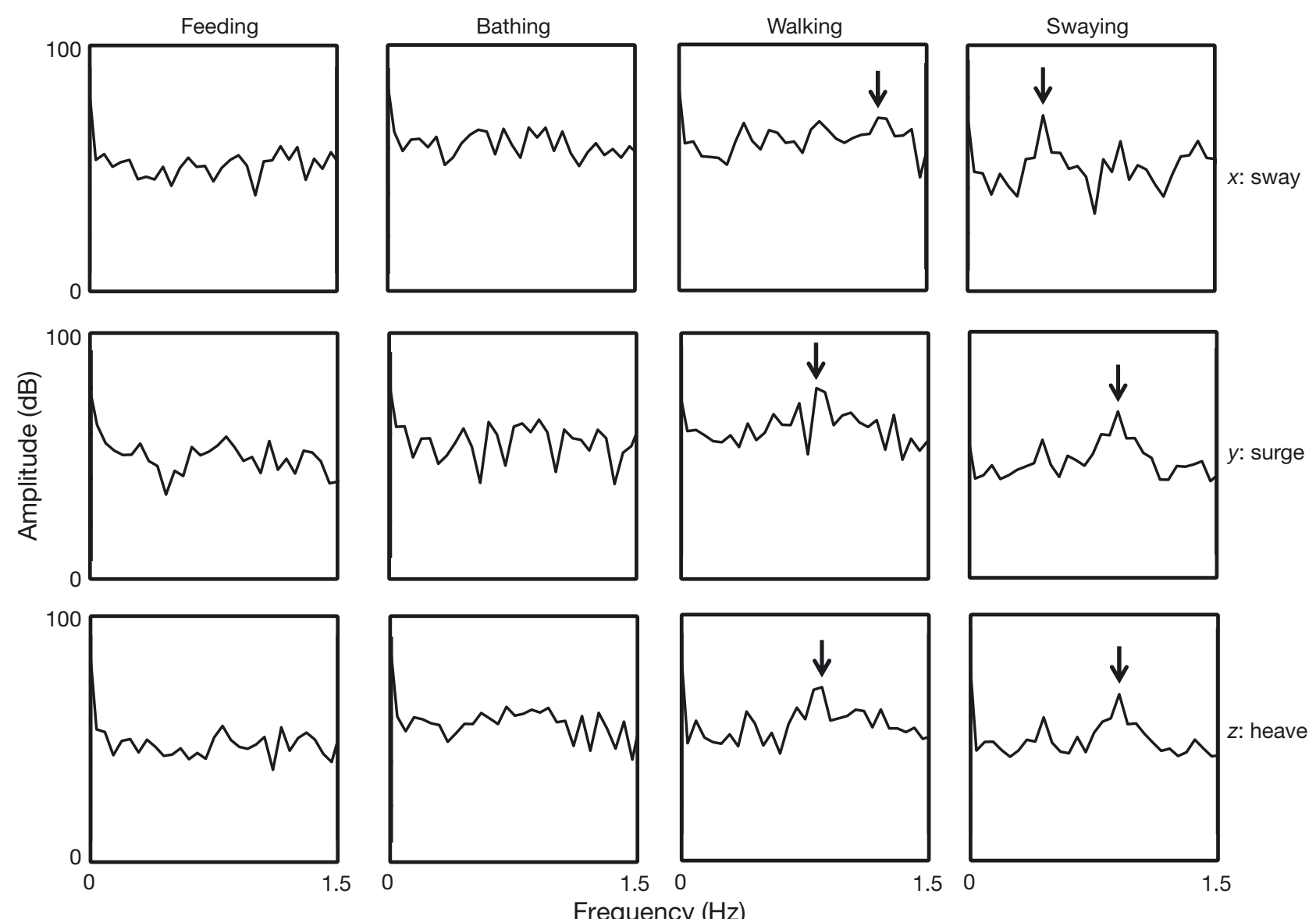

Fig. 3. Loxodonta africana. Fourier transformations of accelerometer data for 4 behaviors from 1 adult female (original data from Fig. 2). Scales for all graphs are the same. Feeding and bathing data exhibit little evidence of periodicity, showing relatively flat spectra with few or no prominent spectral peaks. Walking and swaying spectra exhibit evidence of periodicity in the form of prominent spectral peaks. For periodic behaviors, arrows indicate peak frequencies. For walking, there is no strong evidence for spectral peaks in the sway axis, but the most prominent peak is located at $1.22 \mathrm{~Hz}$ (1 cycle every $0.82 \mathrm{~s}$ ). Periodicity was stronger in the surge and heave axes, with peaks at about $0.82 \mathrm{~Hz}$ in each case ( 1 cycle every $1.22 \mathrm{~s})$. For swaying, periodicity was evident in all 3 axes. Peak frequencies were located at $0.45 \mathrm{~Hz}$ in the sway axis (1 cycle every $2.22 \mathrm{~s}$ ), and at $0.90 \mathrm{~Hz}$ in the surge and heave axes (1 cycle every $1.11 \mathrm{~s}$ )

tive cross-validation analysis, functions are derived from a training dataset and tested on an independent dataset. We ran 3 separate cross-validation classifications, in which functions were derived from 2 subjects (training data) and classification success was tested on the remaining subject.

In addition to the DFA classifications, we also employed a decision tree using simple rules to classify accelerometer data into behavioral states (e.g. Gómez-Laich et al. 2008, Wilson et al. 2008). We chose a $1 \mathrm{~h}$ behavioral sequence consisting of $50 \mathrm{~min}$ in the outdoor enclosure and $10 \mathrm{~min}$ in the barn (to include swaying behavior). For this analysis, we included resting behavior (standing still while not engaged in any of the other 4 behaviors defined above), because subjects in the continuous behavioral sequence sometimes stood still while not engaged in other defined behaviors. The rules for classification were based on the range of variation observed across variables used to measure accelerometer data in the initial analysis outlined above, and on 3 min of resting data from 1 subject. The $1 \mathrm{~h}$ accelerometer data sequence was spliced into $20 \mathrm{~s}$ sequences, after which each sequence was classified into a behavioral state based on the decision tree rules. In this way, we produced an activity budget (percent of time engaged in each behavior), and compared that to an activity budget based on direct observation of the behavior from video.

\section{RESULTS}

Descriptive statistics on the untransformed accelerometer data for each of the 4 behaviors are shown in Table 1 . The 12 variables were reduced to 3 factors 
Table 1. Loxodonta africana. Accelerometer data descriptive statistics (mean \pm SD)

\begin{tabular}{|lcccc|}
\hline Axis and measure & Feeding & Bathing & Walking & Swaying \\
\hline$x$ dynamic acceleration $(g)$ & $0.028 \pm 0.010$ & $0.073 \pm 0.042$ & $0.091 \pm 0.027$ & $0.130 \pm 0.012$ \\
$y$ dynamic acceleration $(g)$ & $0.023 \pm 0.008$ & $0.061 \pm 0.027$ & $0.084 \pm 0.025$ & $0.089 \pm 0.008$ \\
$z$ dynamic acceleration $(g)$ & $0.018 \pm 0.005$ & $0.039 \pm 0.022$ & $0.053 \pm 0.013$ & $0.070 \pm 0.008$ \\
$x$ maximum amplitude (dB) & $58.2 \pm 3.6$ & $69.0 \pm 4.3$ & $72.5 \pm 2.7$ & $69.5 \pm 2.0$ \\
y maximum amplitude (dB) & $57.9 \pm 4.1$ & $67.2 \pm 3.5$ & $73.4 \pm 3.4$ & $66.4 \pm 1.3$ \\
z maximum amplitude (dB) & $54.9 \pm 3.3$ & $61.3 \pm 4.3$ & $68.1 \pm 2.8$ & $64.5 \pm 1.4$ \\
$x$ amplitude SD (dB) & $2.78 \pm 0.82$ & $3.39 \pm 0.89$ & $5.24 \pm 1.34$ & $6.65 \pm 1.74$ \\
y amplitude SD (dB) & $4.07 \pm 1.08$ & $4.07 \pm 1.18$ & $6.39 \pm 1.35$ & $7.44 \pm 1.03$ \\
$z$ amplitude SD (dB) & $3.22 \pm 1.27$ & $2.78 \pm 0.72$ & $5.65 \pm 1.53$ & $7.26 \pm 1.17$ \\
x peak frequency (Hz) & $0.981 \pm 0.335$ & $0.690 \pm 0.224$ & $0.661 \pm 0.370$ & $0.446 \pm 0.014$ \\
$y$ peak frequency (Hz) & $0.731 \pm 0.166$ & $0.662 \pm 0.137$ & $0.825 \pm 0.076$ & $0.884 \pm 0.033$ \\
$z$ peak frequency (Hz) & $1.02 \pm 0.217$ & $1.08 \pm 0.260$ & $0.827 \pm 0.173$ & $0.889 \pm 0.028$ \\
\hline
\end{tabular}

in the PCA, explaining $78.5 \%$ of the variance. The first factor related to periodicity in all 3 axes, dynamic acceleration in the sway and surge axes, and the absolute period of movement in the surge axis; the second factor related to the absolute period of movement in the sway axis; and the third factor related to dynamic acceleration and the absolute period of movement in the heave axis. These 3 factors were entered into the DFA in place of the original 12 variables. The first through third functions of the DFA were statistically significant (Wilks' lambda $=0.045$, $\mathrm{df}=9, \mathrm{p}<0.001$ ).

In the leave-one-out classification, the DFA successfully categorized behavior patterns using these accelerometer measurements (Table 2). Overall, the leave-one-out classification was $90.8 \%$ successful at classifying unlabeled behavioral sequences into the correct behavioral category. The expected classification success based on chance was $25 \%$. Not all behaviors were as easily classified as others. Feeding, walking, and swaying behaviors were rarely misclassified with each other. However, bathing behavior was sometimes confused with feeding and walking (Table 2).

In the leave-one-out classification, training and test data were from the same individuals. In the more conservative cross-validation tests, we derived functions based on 2 subjects at a time, and in each case

Table 2. Loxodonta africana. Confusion matrix from the leave-one-out classification. Number (\%) of trials categorized correctly are shown in the bold diagonal

\begin{tabular}{|c|c|c|c|c|}
\hline & Feeding & Bathing & Walking & Swaying \\
\hline Feeding & $27(90)$ & $3(10)$ & 0 & 0 \\
\hline Bathing & $3(10)$ & $26(87)$ & $1(3)$ & 0 \\
\hline Walking & 0 & $3(10)$ & 26 (87) & $1(3)$ \\
\hline Swaying & 0 & 0 & 0 & $30(100)$ \\
\hline
\end{tabular}

tested classification success on the remaining subject ( 3 tests: Wilks' lambda $=0.086,0.085,0.064 ;$ all $\mathrm{df}=6$; all $p<0.001)$. Since swaying was only observed in 1 individual, cross-validation tests only included feeding, bathing, and walking behaviors. The average classification success across the 3 tests was $87.8 \%$. The expected classification success based on chance was $33.3 \%$. As in the leave-one-out classification, walking and feeding were easily distinguished from each other (each with an average of $90 \%$ classification success), while bathing showed a lower success rate of $83.3 \%$.

Descriptive statistics from the foregoing analyses were used to construct a simpler decision tree that may be used for practical purposes (Fig. 4). We applied the decision tree rules to a 1-h continuous

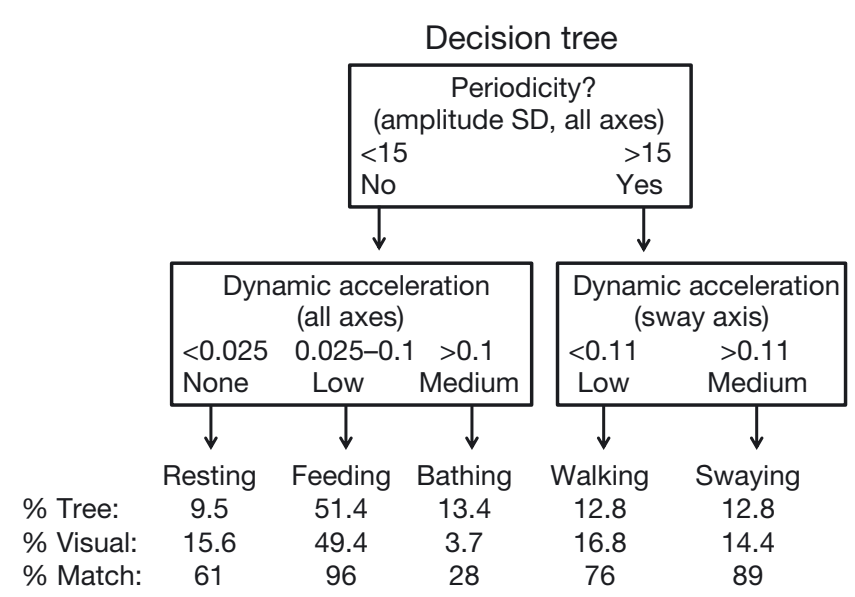

Fig. 4. Loxodonta africana. Decision tree for classifying a $1 \mathrm{~h}$ stream of accelerometer data into behavioral states. \% Tree: percentage of time spent in each behavioral state based on the decision tree analysis; \% Visual: percentage of time spent in each behavioral state based on visual observation of behavior from video; \% Match: percent match between the 2 methods ([lowest percent / highest percent] $\times 100$ ) 
stream of accelerometer data, and compared the activity budget produced by the decision tree to that produced by visual observation of the video. Overall, the decision tree matched the visual observation with $70 \%$ accuracy. As in the previous classification analyses, the accuracy was relatively high for feeding $(96 \%)$, swaying $(89 \%)$, and walking $(76 \%)$, but was very low for bathing $(28 \%)$.

\section{DISCUSSION}

\section{Summary of results}

Data from tri-axial accelerometers placed on the top of elephants' necks successfully identified elephant behaviors, when combining measures of overall dynamic acceleration with measures of periodicity (for similar examples in cats and birds, see Watanabe et al. 2005 and Sakamoto et al. 2009). When training and test data were from the same individuals (leaveone-out classification), a high overall success rate was achieved (91\%). This result suggests that accelerometers can in principle be highly effective behavioral monitoring devices whenever validation tests can be run on individual elephants, such as in zoos. Even in the more conservative cross-validation tests, in which training data came from 2 subjects and were tested on the remaining subject, the overall classification success was high (88\%). Although further work will be needed (see 'Caveats and future directions' below), this result suggests that accelerometer measurements on a subset of elephants may be used to measure behavior of individuals that have not been tested previously; this finding makes the application rather promising for wild elephants. Even a simple decision tree showed modest overall success (70\%) at identifying behaviors from a continuous stream of accelerometer data, compared to direct observation of the behavior from video. Thus, accelerometers may be a useful tool for remotely measuring behavior of elephants wherever they are found.

\section{Interpretation of results}

Taken together, the validation tests showed that elephant behaviors are detectable using accelerometers, but some behaviors were more readily distinguished than others. In general, swaying, walking, and feeding were the most easily differentiated, while bathing was often confused with feeding and walking. Examination of the dynamic acceleration and periodicity recorded for each of the behaviors will shed light on the variable classification success observed.

Feeding behavior exhibited very low dynamic acceleration in all 3 axes, and little evidence of periodicity of movement in any axis (Figs. 2 \& 3). Searching for food from the ground with the trunk and lifting of the trunk to the mouth at irregular intervals did not result in large movements of the neck and this activity was not cyclic in nature. As such, feeding was easily distinguished from the other behaviors (Table 2), which exhibited either relatively high levels of dynamic acceleration (bathing, walking, and swaying) or greater periodicity of movement (walking and swaying).

Bathing behavior exhibited intermediate levels of dynamic acceleration and little evidence of periodicity of movement (Figs. $2 \& 3$ ). This reflected the low levels of neck movement while the elephant was using the trunk to acquire mud or water from ground level, and the greater levels of acceleration when the trunk was used to propel the water or mud onto various parts of the body (i.e. under the body, over the top of the body, and to the sides of the body). This resulted in irregularly spaced spikes in dynamic acceleration in all 3 axes, but little periodicity of movement. Bathing behavior was sometimes confused with other behaviors, in particular with feeding and walking (Table 2). Bathing was confused with feeding because of the structural similarity of using the trunk to acquire food from the ground and using it to acquire water or mud at ground level, at which times the 2 behaviors would resemble each other. Bathing was confused with walking when the walking gait was not rhythmic enough to detect periodicity (for example, when the elephant was beginning to walk or slowing to stop), at which times the lack of periodicity and the relatively high acceleration resembled bathing.

Walking behavior was characterized by variable, but generally high dynamic acceleration and by variable, but generally high periodicity of movement (Figs. 2 \& 3). The observed variation was probably due to varying speeds of walking, in which slower walking speeds resulted in less dynamic acceleration and less periodicity of movement. Periodicity of movement was apparent in all 3 axes, but was greatest in terms of surge (front-back movement) and heave (up-down movement), as the head moved mainly up and down and backward and forward during locomotion. The cycle frequency in the surge and heave axes was about 1 cycle per $1.22 \mathrm{~s}$, reflecting the gait of the elephants in these observations. 
Swaying behavior was characterized by consistently high dynamic acceleration and high periodicity of movement (Figs. $2 \& 3$ ). This consistency was due to the highly stereotypical nature of this behavior. In the one individual for which we obtained swaying data, the dominant motion was lateral swaying. This motion produced relatively high dynamic acceleration and periodic acceleration in the sway axis, with a cycle frequency of about 1 cycle per $2.2 \mathrm{~s}$. Swaying simultaneously produced high levels of dynamic and periodic acceleration in the surge axis (front-back movement), as the head moved in an arc during each swing, exerting outward forces, and in the heave axis (up-down movement), as the elephant dipped her head at the beginning and end of each lateral swing. The period of movement in the surge and heave axis was about 1.1 cycles s $^{-1}$, about half of the period of the sway axis, as the surge arcs and heave dips occurred twice per lateral swing. Swaying was distinguished from walking mainly in the sway axis, the former exhibiting greater dynamic acceleration and a longer cycle frequency (Figs. $2 \& 3$ ).

In a more realistic analysis, we also processed a continuous 1-h accelerometer data stream. For this analysis, we used a simple decision tree (Fig. 4) based on descriptive statistics in the original analyses (Table 1). The activity budget based on the decision tree generally matched the activity budget based on direct observation of behavior (Fig. 4), but not all behaviors were equally distinguishable. As the previous analyses showed, accelerometer data most effectively distinguished feeding, walking, and swaying behaviors, but not bathing behavior.

\section{Caveats and future directions}

The combination of standard measures of dynamic acceleration and of periodicity successfully distinguished between elephant behavior patterns. Further experimentation will determine which specific data analysis methods are best for distinguishing elephant behaviors, both in terms of complex statistical analyses and the simpler decision tree rules. For example, hidden Markov models achieved a higher classification success rate compared to methods similar to those employed here, when categorizing elephant vocalizations by individual identity (see Clemins et al. 2005, Soltis et al. 2005). For many practical applications, however, such intensive statistical analyses will not be possible. Thus, refinement of simple rules of thumb, such as those described in Fig. 4, will be needed if accelerometers are to prove useful in applied settings.
Moving forward, analyses will have to be conducted on more individuals and ideally also across the 2 elephant species. In our cross-validation analysis, we showed that accelerometer data associated with feeding, bathing, and walking behavior in 1 elephant could be used to successfully identify the same behavioral states in another elephant, indicating that those specific behavioral patterns did not vary too greatly across individuals. However, sample sizes will have to be increased to determine the precise measurements that are robust enough to generalize across a larger number of individuals. This is also true of swaying, which as noted was measured in only 1 animal, because even 'stereotypical' behaviors like swaying are likely to differ in detail across individual elephants. For example, swaying behavior may be predominantly side-to-side, as in this case, but can also occur in a front-to-back motion (e.g. Gruber et al. 2000). It should also be emphasized that this work was conducted on adult females, so this process should be repeated for other age and sex classes as well.

Only 5 behavior patterns were examined in the analyses reported here (including resting), so other behaviors will need to be added in future analyses. As other behaviors are added to such analyses, however, behaviors may become less distinguishable from one another using accelerometers (e.g. see Martiskainen et al. 2009). Also, the 5 behaviors were chosen because they occur in relatively long bouts so that many $20 \mathrm{~s}$ sequences could be obtained for analysis. Many other elephant behaviors are shortlived, however, such as head-shaking and rubbing the body against substrates. It is unclear whether such short-lived behaviors will be amenable to timeseries analyses in the same way that more long-lived behaviors are.

Finally, to be of use to many professionals working with elephants, the process of measurement and classification will have to be made robust, as noted above, and then automated. At that point, the analysis of behavior could be made in real time (with wireless download) or at least immediately upon retrieval of the accelerometer (without wireless download). If the technique is taken this far, it could have widespread use for elephants worldwide. In zoos, accelerometers could be used to compile daily reports of elephant activity levels and behavioral profiles for staff veterinarians and elephant managers. In the wild, accelerometers combined with GPS units could detect when elephants are in various types of distress or danger, and send electronic messages to appropriate personnel, such as wildlife veterinarians and antipoaching units (see 'Introduction' for more details). 
The preliminary results presented here indicate that accelerometers attached to existing and widely used elephant collars can distinguish between elephant behaviors, and with further development can be deployed as powerful tools for elephant care in both wild and captive contexts.

Acknowledgements. The project was implemented by the Disney's Animal Kingdom ${ }^{\circledR}$ Elephant Team and the Education and Science Team. We thank the members of both teams who assisted in designing the accelerometer housing, for conducting the collaring and de-collaring of elephants, and for assistance in data collection and analysis. We also thank 2 reviewers for very helpful comments on a previous version of this paper. This research was approved by Disney's Animal Care and Welfare Committee.

\section{LITERATURE CITED}

Barthélémy I, Barrey E, Thibaud JL, Uriarte A, Voit T, Blot S, Hogrel JV (2009) Gait analysis using accelerometry in dystrophin-deficient dogs. Neuromuscul Disord 19:788-796

Boersma P, Weenink D (2007) PRAAT: Doing phonetics by computer (Version 4.5.18). Available at www.praat.org/ (accessed April 2007)

> Chapinal N, de Passillé AM, Pastell M, Hänninen L, Munksgaard L, Rushen J (2011) Measurement of acceleration while walking as an automated method for gait assessment in dairy cattle. J Dairy Sci 94:2895-2901

- Clemins PJ, Johnson MT, Leong KM, Savage A (2005) Automatic classification and speaker identification of African elephant (Loxodonta africana) vocalizations. J Acoust Soc Am 117:956-963

Enstipp MR, Ciccione S, Gineste B, Milbergue M and others (2011) Energy expenditure of freely swimming adult green sea turtles (Chelonia mydas) and its link with body acceleration. J Exp Biol 214:4010-4020

Gage LJ (2001) Treatment of osteomyelitis in elephant feet. In: Csuti B, Sargent EL, Bechert US (eds) The elephant's foot. Iowa State University Press, Ames, IA, p 117-118

> Godfrey A, Conway R, Meagher D, ÓLaighin G (2008) Direct measurement of human movement by accelerometry. Med Eng Phys 30:1364-1386

> Gómez Laich A, Wilson RP, Quintana F, Shepard ELC (2008) Identification of imperial cormorant Phalacrocorax atriceps behaviour using accelerometers. Endang Species Res 10:29-37

- Gruber TM, Friend TH, Gardner JM, Packard JM, Beaver B, Bushong D (2000) Variation in stereotypic behavior related to restraint in circus elephants. Zoo Biol 19: 209-221

> Halsey LG, Green JA, Wilson RP, Frappell PB (2009) Accelerometry to estimate energy expenditure during activity: best practices with data loggers. Physiol Biochem Zool 82:396-404

Leighty KA, Soltis J, Wesolek CM, Savage A (2008) Rumble vocalizations mediate interpartner distance in African elephants, Loxodonta africana. Anim Behav 76:1601-1608

Leighty KA, Soltis J, Wesolek CM, Savage A, Mellen J, Lehnhardt J (2009) GPS determination of walking rates in captive African elephants (Loxodonta africana). Zoo Biol 28:16-28

Editorial responsibility: Jonathan Green, Liverpool, UK
Lyons GM, Culhane KM, Hilton D, Grace PA, Lyons D (2005) A description of an accelerometer-based mobility monitoring technique. Med Eng Phys 27:497-504

Martiskainen P, Järvinen M, Skón JP, Tiirikainen J, Kolehmainen M, Mononen J (2009) Cow behaviour pattern recognition using a three-dimensional accelerometer and support vector machines. Appl Anim Behav Sci 119:32-38

> Moreau M, Siebert S, Buerkert A, Schlecht E (2009) Use of a tri-axial accelerometer for automated recording and classification of goats' grazing behaviour. Appl Anim Behav Sci 119:158-170

> Okuyama J, Kawabata Y, Naito Y, Arai N, Kobayashi M (2009) Monitoring beak movements with an acceleration datalogger: a useful technique for assessing the feeding and breathing behaviors of sea turtles. Endang Species Res 10:39-45

Quinn GP, Keough MJ (2002) Experimental design and data analysis for biologists. Cambridge University Press, Cambridge

Ringgenberg N, Bergeron R, Devillers N (2010) Validation of accelerometers to automatically record sow postures and stepping behaviour. Appl Anim Behav Sci 128: 37-44

Rothwell ES, Bercovitch FB, Andrews JRM, Anderson MJ (2011) Estimating daily walking distance of captive African elephants using an accelerometer. Zoo Biol 30: $579-591$

Sakamoto KQ, Sato K, Ishizuka M, Watanuki Y, Takahashi A, Daunt F, Wanless S (2009) Can ethograms be automatically generated using body acceleration data from freeranging birds? PLoS ONE 4:e5379

Sala JE, Quintana F, Wilson RP, Dignani J, Lewis MN, Campagna C (2011) Pitching a new angle on elephant seal dive patterns. Polar Biol 34:1197-1209

Shepard ELC, Wilson RP, Quintana F, Gómez Laich A and others (2008) Identification of animal movement patterns using tri-axial accelerometry. Endang Species Res 10: 47-60

Soltis J, Leong K, Savage A (2005) African elephant vocal communication. II: Rumble variation reflects individual identity and emotional state of callers. Anim Behav 70: 589-599

Soltis J, Blowers TE, Savage A (2011) Measuring positive and negative affect in the voiced sounds of African elephants (Loxodonta africana). J Acoust Soc Am 129: 1059-1066

> Wall J, Douglas-Hamilton I, Vollrath F (2006) Elephants avoid costly mountaineering. Curr Biol 16:R527-R529

Watanabe S, Izawa M, Kato A, Ropert-Coudert Y, Naito Y (2005) A new technique for monitoring the detailed behaviour of terrestrial animals: a case study with the domestic cat. Appl Anim Behav Sci 94:117-131

Whitney NM, Pratt HL Jr, Pratt TC, Carrier JC (2010) Identifying shark mating behavior using three-dimensional acceleration loggers. Endang Species Res 10:71-82

Wilson RP, White CR, Quintana F, Halsey LG, Liebsch N, Martin GR, Butler PJ (2006) Moving towards acceleration for estimates of activity-specific metabolic rate in freeliving animals: the case of the cormorant. J Anim Ecol 75: 1081-1090

Wilson RP, Shepard ELC, Liebsch N (2008) Prying into the intimate details of animal lives: use of a daily diary on animals. Endang Species Res 4:123-137 